\title{
Pigeons learn to answer the question "where did you just peck?" and can report peck location when unexpectedly asked
}

\author{
Rebecca A. Singer And Thomas R. Zentall \\ University of Kentucky, Lexington, Kentucky
}

\begin{abstract}
If pigeons are trained on matching-to-sample with differential responding required to the two samples, there is evidence that the differential responding can control comparison choice. We asked whether similar responding required at two different locations could also serve as the basis for comparison choice. Pigeons were pretrained to report the location that they had pecked. To reduce the likelihood that they could use the presence of differential proprioceptive cues at the time of their report, a common response was required between the location response and the comparison choice. They were then given experience with a conditional discrimination in which location of the comparison response varied randomly and was incidental to the choice of comparison. On test trials, after the pigeons had made their comparison choice, they showed a significant tendency to choose the appropriate test comparison when they were unexpectedly asked to report the location of their previous pecking response. These results have implications for the demonstration of episodic-like memory in pigeons because they suggest that pigeons have the capacity to recall, unexpectedly, specific details about their past experiences.
\end{abstract}

Symbolic matching-to-sample typically involves two sample or conditional stimuli (e.g., vertical or horizontal lines) and two comparison or test stimuli (e.g., red and green hues). When pigeons are trained on such a task, they have to learn, for example, to choose red when the sample is vertical lines and to choose green when the sample is horizontal lines. Usually, the samples are presented for a fixed duration (Blough, 1959), or a fixed number of responses are required to the sample (Zentall \& Hogan, 1974), after which the comparison stimuli appear. However, when differential responding is required to the two samples, there is evidence that the two differential sample responses themselves exert significant control over comparison choice (Urcuioli \& Honig, 1980). For example, Urcuioli and Honig (Experiment 3) trained pigeons to respond differentially to vertical and horizontal line orientation samples in a matching task. In the presence of one of the samples, they had to peck 10 times (fixed ratio [FR] 10) to obtain the comparison stimuli. In the presence of the other sample, they had to space two responses $3 \mathrm{sec}$ apart (differential reinforcement of a low rate of pecking [DRL] $\left.3^{\prime \prime}\right)$. They then trained the pigeons to make the same differential response (FR 10 and DRL 3") to two different stimuli (red and green hues, respectively) in a simple successive discrimination. On transfer trials, when Urcuioli and Honig replaced the vertical and horizontal line orientation samples with the red and green hues, they found that the pigeons showed a high degree of transfer. The pigeons chose the comparison that, in training, had been as- sociated with the appropriate sample response about $85 \%$ of the time, despite the fact that they had never before seen those hues as samples with the line comparisons.

In a typical matching task, one can view the presentation of the comparison stimuli as analogous to asking the pigeon, "What sample did you just see?" But when differential sample responding is required and it appears to control comparison choice, the question would become, "What did you just do (e.g., peck quickly or peck slowly)?"

In a related experiment, Zentall, Clement, Bhatt, and Allen (2001) first trained pigeons to match vertical and horizontal line samples to red and green comparison stimuli, with differential responding required to the two samples. Specifically, when the samples consisted of vertical lines, the first response after $4 \mathrm{sec}$ produced the comparison stimuli, and the red comparison stimulus was correct. This requirement resulted in a high rate of pecking the vertical lines. When the samples consisted of horizontal lines, the pigeons had to refrain from pecking for $4 \mathrm{sec}$ to produce the comparison stimuli, and the green comparison stimulus was correct. The pigeons quickly learned to refrain from pecking the horizontal lines. One way to view this task is from the perspective of the comparison stimuli. When they are presented, they are analogous to asking the question, "What did you just do?" If the pigeons had just pecked, they should choose the red comparison stimulus. If they had just refrained from pecking, they should choose the green comparison stimulus.

In Phase 2, Zentall et al. (2001) trained the pigeons on a differential autoshaping task in which, on some trials,

T. R.Zentall, zentall@uky.edu 
a yellow stimulus was presented for $4 \mathrm{sec}$ and then food appeared. On other trials, a blue stimulus was presented for $4 \mathrm{sec}$, but it was not followed by food. Although food was presented noncontingently following presentation of the yellow stimulus, the pigeons would typically peck at it, whereas they would typically refrain from pecking at the blue stimulus.

On test trials, the pigeons were presented with yellow and blue stimuli as samples, followed by red and green comparison stimuli, and positive transfer resulted. That is, when the sample was the yellow stimulus that they had pecked because it had been followed by food, the pigeons tended to choose the comparison that, in original training, had been associated with sample pecking, whereas when the sample was the blue stimulus that they had refrained from pecking, they tended to peck the comparison that, in original training, had been associated with the absence of pecking (see also Urcuioli \& DeMarse, 1994).

If comparison presentation following yellow and blue samples can be thought of as analogous to asking the question, "What did you just do?" how does the pigeon answer that question? On original training trials, the pigeon can learn to anticipate presentation of the comparison stimuli and prepare to choose the correct comparison stimulus. On test trials involving the yellow and blue stimuli from differential autoshaping, however, there should be no expectation of the appearance of the red and green comparison stimuli. That is, on test trials, the appearance of the comparison stimuli should be surprising, and the pigeon should have to recall or refer back to the response it made to the initial stimulus that had been presented before the comparison stimuli appeared. Referring back to an earlier behavior can be thought of as an episodic-like memory. The question of how one should define an episodic-like memory will be discussed later in more detail. For now, however, it is sufficient to assume that on test trials, there are no residual motor aftereffects (proprioceptive cues) present at the time the comparison stimuli are presented to indicate which comparison stimulus should be chosen. However, when the behavior to be retrieved is responding versus refraining from responding, it is possible that the aftereffects of responding (e.g., a sensation in the beak or neck) may persist for a short time, long enough to serve as a cue for comparison choice.

The purpose of the present experiment was to use a different kind of differential-sample response, the location of the response. In this case, whatever residual motor aftereffects are present should not be differential and, thus, should not cue comparison choice. In the present experiment, in Phase 1, we asked pigeons to report the location (left or right) of a previous pecking experience to otherwise undifferentiated white pecking keys by choosing a red or a green comparison stimulus. To avoid allowing the pigeon to use the position of its beak at the time of the appearance of the comparison stimuli, on every trial, following the left or right initial response, it was required to peck a triangle at a common location (on the center key). The common response also created a delay interval between the left or right pecking behavior and presentation of the comparison stimuli, and the delay interval should have increased the necessity for retrieval from memory. The pigeons were then trained on a symbolic-matching task to choose vertical lines when the sample was a blue hue and to choose horizontal lines when the sample was a yellow hue. Because the vertical and horizontal lines appeared randomly on the left and right response keys, pecking at the different locations was an incidental aspect of this task. Finally, the pigeons were tested on probe trials on which, after the choice of line orientation, the pigeons received a triangle on the center key, followed by a choice of red and green comparison stimuli. Thus, on these probe trials, the pigeons were unexpectedly asked which side key they had last pecked.

\section{METHOD}

\section{Subjects}

The subjects were 8 White Carneau pigeons (Columba livia), retired breeders, purchased from the Palmetto Pigeon Plant (Sumter, SC). They were maintained at $85 \%$ of their free-feeding body weight for the duration of the experiment, were caged individually with free access to grit and water in the home cage, and were cared for in accordance with University of Kentucky animal care guidelines. The colony room was maintained on a 12:12-h light:dark cycle. All the pigeons had previous experience with simple simultaneous discriminations.

\section{Apparatus}

The experiment was conducted in a BRS/LVE (Laurel, MD) sound-attenuating pigeon test chamber. Three round response keys (2.5- $\mathrm{cm}$ diameter) were aligned horizontally on the response panel. A 12-stimulus in-line projector (Industrial Electronics Engineering, Van Nuys, CA) with 28 V, 0.1 A lamps (GE 1820) was mounted behind each response key. The center response key projected blue, yellow, red, and green hues (Kodak Wratten Filter Nos. 38, 9, 26, and 60 , respectively) and a small solid white equilateral triangle on a black background. The left and right response keys projected white (unfiltered), red, and green hues, as well as three white vertical lines and three white horizontal lines on a black background. A houselight located at the center of the chamber ceiling provided general illumination during intertrial intervals. A rear-mounted grain feeder provided mixed grain reinforcement (Purina Pro Grains) through an aperture centered horizontally on the response panel. Reinforcement consisted of 2-sec access to mixed grain. An exhaust fan mounted on the outside of the chamber masked extraneous noise. The experiment was controlled and data collected by a microcomputer located in the adjacent room.

\section{Procedure}

Phase 1. Phase 1 consisted of conditional discrimination training. Each trial began with the onset of a white stimulus on the left or right side key. Five responses to the white key were followed by the presentation of a white triangle on the center key. The pigeons were required to peck the triangle once to turn it off and turn on the red and green comparison stimuli on the two side keys. For half of the pigeons, choice of the red comparison stimulus was rewarded following the left white side key and choice of the green comparison stimulus was rewarded following the right white side key (see top panel of Figure 1). For the remaining pigeons, the contingencies were reversed. The pigeons were required to peck once at the comparison stimulus. Choice of the correct comparison was rewarded with 2 -sec access to mixed grain, followed by a $10-\mathrm{sec}$ intertrial interval (ITI) during which the houselight was lit. Choice of the incorrect comparison was followed by a 10 -sec lit ITI only. The red 
Pigeons Learn to Answer 20
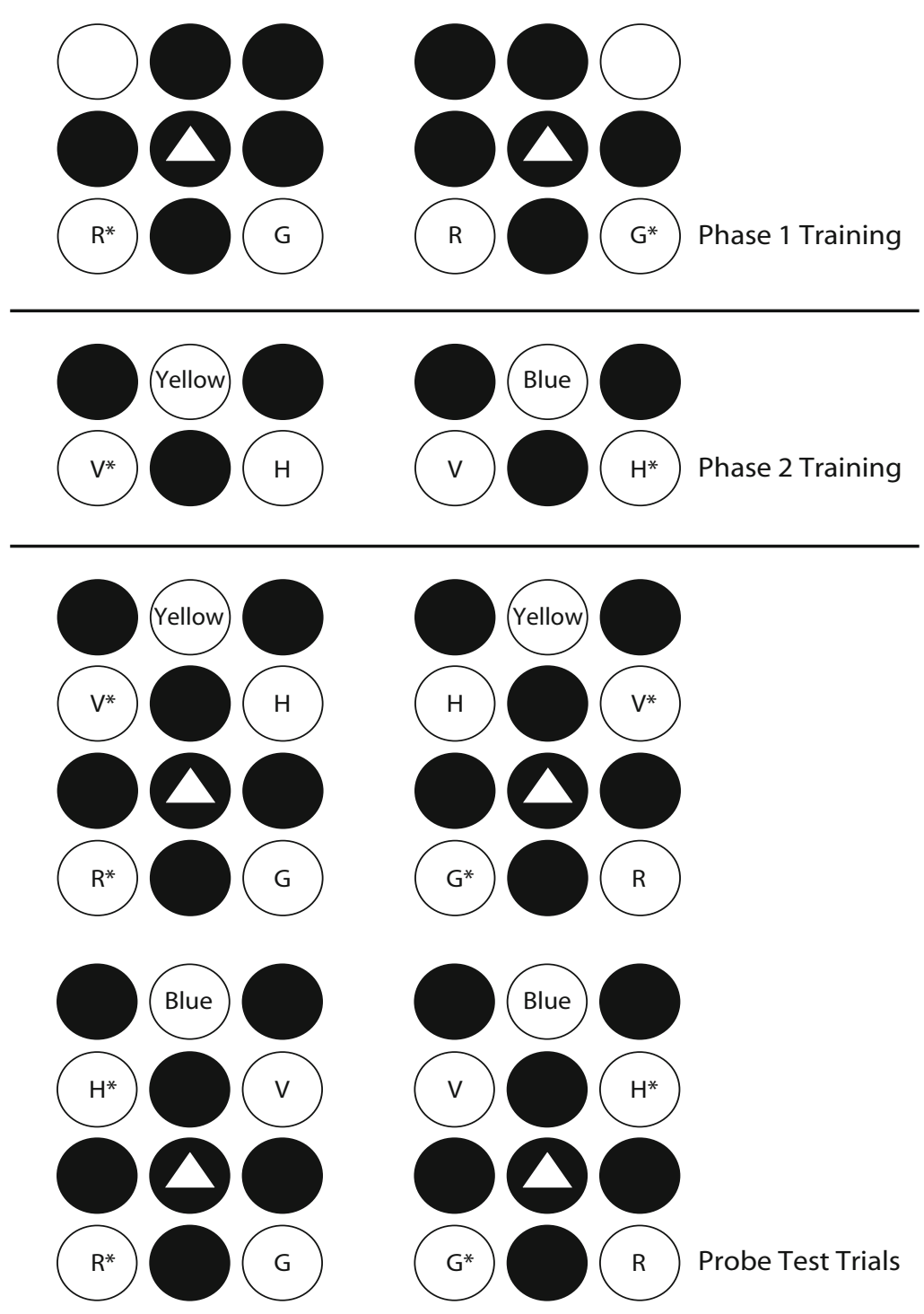

Figure 1. Experimental design. The top panel illustrates the conditional discrimination used in Phase 1 , in which a white side key was followed by a center triangle and then by red and green comparison stimuli. The middle panel shows the conditional discrimination used in Phase 2 , in which yellow and blue samples were followed by vertical and horizontal line comparison stimuli, with comparison location (left and right) counterbalanced. The bottom panel illustrates the four probe test trial types in which a Phase 2 conditional discrimination was followed by the center triangle stimulus and then by red and green comparison stimuli, with comparison location (left and right) counterbalanced. In Phases 1 and 2, an asterisk indicates which comparison choice was correct and, on probe test trials, which comparison choice was consistent with retrieval of the spatial location acquired in Phase 1. On probe test trials, all choices were reinforced $50 \%$ of the time.

and green comparison stimuli served to ask the question, "Where did you last peck a side key, on the left or on the right?"

All the pigeons received 96 trials per session with equal numbers of trials initiated by the right and left side keys. Sessions were conducted 6 days a week. The pigeons remained in Phase 1 until they reached a criterion of $90 \%$ correct on trial types initiated by each of the two side keys for two consecutive sessions.
Phase 2. Phase 2 consisted of symbolic matching-to-sample training in which color samples (blue and yellow) were followed by line orientation comparisons (vertical and horizontal). Each trial began with the presentation of a yellow or blue stimulus on the center response key. After the pigeon had pecked the sample stimulus 10 times, the vertical and horizontal comparison stimuli were presented on the side keys. Five pecks were required to the line orientation 
"Where Did You Just Peck?"

comparisons. For half of the pigeons, vertical lines were correct following a yellow sample, and horizontal lines were correct following a blue sample (see the middle panel of Figure 1). The contingencies were reversed for the remaining pigeons.

Each session consisted of 96 trials, with an equal number of trials initiated by blue and yellow samples. The first 72 trials were symbolic matching-to-sample trials. The last 24 trials served as refresher trials and were identical to Phase 1 trials. The pigeons were trained until they reached a criterion of $90 \%$ correct on all four trial types for 2 consecutive days. The pigeons then received training with the Phase 1 and Phase 2 trials randomly mixed until they regained the criterion of $90 \%$ correct on all four trial types for 2 consecutive days.

Probe trials. During testing there were 104 trials per test session: 48 Phase 1 trials, 48 Phase 2 trials, and 8 probe trials. On probe trials, yellow and blue hues were presented on the center response key, followed by vertical and horizontal line comparisons (as in Phase 2); however, comparison choice was followed by presentation of the triangle on the center key, and a single peck to the triangle was followed by the red and green comparison stimuli (see the bottom panel of Figure 1). Choice of red and green comparisons was rewarded nondifferentially ( $50 \%$ of the time). Testing involved a total of 24 sessions.

\section{RESULTS}

\section{Training}

It took pigeons between 6 and 41 sessions to reach criterion in Phase $1(M=18.25, S E M=4.519)$ and between 25 and 90 sessions to reach criterion in Phase $2(M=$ 55.87, $S E M=9.384)$.

\section{Probe Trials}

On the first test session ( 8 probe trials), the pigeons chose the red or green hue that was appropriate for the location of the previous line orientation choice $67.2 \%$ of the time. A one-sample, two-tailed $t$ test performed on the probe trial data from Session 1 indicated that this was significantly above chance $[t(7)=2.31, p=.05]$. Over all probe trials, the pigeons correctly reported where they had just pecked $71.6 \%$ of the time $[t(7)=10.73, p<.001]$. Thus, despite the absence of differential reinforcement, there was apparently little change in performance over sessions on the infrequently presented probe trials. The data from Session 1 and pooled over test sessions appear in Table 1.

On test trials, the pigeons pecked a line orientation on the left or right response key, followed by a peck to the triangle on the center key, followed by choice of the red or green side key. Because we were interested in the pigeons' ability to retrieve the memory of the location previously pecked, we measured the time between the initial side key response and choice of red or green side key for the pigeons. On average, the time between the left/right location response and the pigeons' red/green choice response was $1.74 \mathrm{sec}$ (with a range of 1.05-2.51 sec).

\section{DISCUSSION}

When the pigeons were unexpectedly asked to recall the location that they had recently pecked, they responded correctly on most trials. These results demonstrate that the pigeons were able to retrieve information about their past
Table 1

Choice of the Hue Appropriate for the Location of the Previous Line Orientation Choice on Session 1 and Overall Test Sessions for Each Pigeon

\begin{tabular}{ccc}
\hline $\begin{array}{c}\text { Pigeon } \\
\text { No. }\end{array}$ & $\begin{array}{c}\text { Session 1 } \\
\text { (No. Correct Out of 8) }\end{array}$ & $\begin{array}{c}\text { Overall } \\
\text { (\% Correct) }\end{array}$ \\
\hline 1 & 6 & 61.5 \\
2 & 7 & 77.1 \\
3 & 8 & 69.3 \\
4 & 5 & 69.3 \\
5 & 4 & 74.5 \\
6 & 6 & 72.9 \\
7 & 4 & 68.8 \\
8 & 3 & 79.7 \\
$M$ & $5.4(67.5 \%)$ & 71.6 \\
\hline
\end{tabular}

experience, although the location of their choice of line orientation had never before been requested. Furthermore, in the context of hue-sample-line-orientation symbolic matching, the location of the correct line orientation comparison stimulus was incidental to the task. These results extend the results of Zentall et al. (2001) from pecking versus the absence of pecking to pecking left versus pecking right. Furthermore, to the extent that the residual motor aftereffects of pecking the center are likely to be quite similar, as compared with pecking versus refraining from pecking in Zentall et al.'s study, the present experiment also makes it less likely that the pigeons were using such possible aftereffects as the basis for comparison choice.

The ability to respond correctly to the unexpected question, "Where did you peck?" suggests that the pigeons were able to retrieve information about a past experience. Such an ability can be thought of as similar to having an episodic memory. Episodic memory is a form of declarative memory that can be distinguished from semantic or rule-based memory. Semantic memories consist of factual information, such as the capital of Kentucky is Frankfort, or rules, such as if the white light appeared on the left, choose the red comparison. Episodic memories consist of personal experiences, or what Tulving (1985) called autonoetic memories. In human memory, a classic example of episodic memory is being able to answer the question, "What did you have for breakfast this morning?" Use of the term episodic memory implies a cognitive traveling back in time. Because it is difficult to know by what mechanism pigeons recover personal experiences, we prefer to use the term episodic-like to describe the performance.

There has been some ambiguity over what constitutes human episodic memory. According to Tulving (1972), a person who has episodic memory must be able to identify a past personal experience in terms of what happened, where it happened, and when it happened. Although, since then, Tulving (1985) has added additional cognitive features to his definition, such as self-awareness and autonoetic consciousness, those characteristics are difficult to assess in humans, much less in nonverbal animals. As a result, Clayton and Dickinson (1999a, 1999b) used what they described as Tulving's earlier what, where, and when criterion to establish a behavioral test of episodic-like memory in animals. Clayton and Dickinson allowed scrub jays to 
cache two types of food, wax worms and peanuts (what), in distinctive locations (where). The scrub jays discovered that their preferred food, wax worms, went bad over time but that the less preferred peanuts did not (when). The jays learned to recover the wax worm if relatively little time had passed but to retrieve the peanuts if more time had passed since caching. Thus, it appears that the scrub jays learned about the what, when, and where of a previous food-caching experience.

Babb and Crystal (2006) used a similar approach to study episodic-like memory in rats. They first allowed rats to enter several baited arms of a radial maze, one of which contained a unique chocolate reward. Either later that day or $24 \mathrm{~h}$ later, with all the arms open, they allowed the rats to complete the trial (enter the remaining arms, which were baited), and depending on the condition, the arm that had previously been baited with chocolate was again baited with chocolate or was not. The baited arms, including the chocolate arm, changed location on each trial.

Babb and Crystal (2006) found that the rats were more likely to enter the arm that had contained the chocolate at the appropriate time (either later that day or $24 \mathrm{~h}$ later, depending on when they had learned that the chocolate would be replenished) than at the inappropriate time, indicating that they too had learned about the what, where, and when of a previous experience.

An important question is whether the what-wherewhen criterion for episodic-like memory sufficiently distinguishes it from the other form of declarative memory, semantic (or rule-based) memory, which consists of general knowledge or learned rules. Most memory research with animals is thought to be rule based. A delayed conditional discrimination involves the acquisition of a set of rules (e.g., choose the comparison color that is the same as the sample color). In principle, one could develop a task that would satisfy the what-where-when criterion for pigeons. For example, pigeons might be able to learn to match the color of the sample (red or green) when it appeared on the right response key but to mismatch the color of the sample when it appeared on the left response key. But those rules would apply only if the sample had been presented recently (e.g., within the past $2 \mathrm{sec}$ ). If the sample had appeared $5 \mathrm{sec}$ before, the pigeons would be rewarded for matching the color of the sample when it appeared on the left and for mismatching the color of the sample when it appeared on the right. Although this task would likely be quite difficult for the pigeons to acquire, we do not believe that it corresponds to what is considered episodic memory. Instead, it may consist of eight acquired rules and can be better characterized as a form of knowing (semantic or rule learning).

To better characterize the distinction between episodic and rule-based memory, consider the example of episodic memory given earlier: the answer to the question, "What did you have for breakfast this morning?" That memory is assumed to be episodic because there was no expectation at the time of the meal (or even shortly after the meal) that the question would be asked. But now consider that the question is asked every day. It is quite possible that after several days, the responder would begin to use a language-based rule contingent on the sample of food on the table - a rule such as "remember to say, "toast and coffee." The difference between these two kinds of memory is that when the responder was asked initially, he/she presumably had to mentally "travel back in time" to retrieve the remembered episode, whereas after he/she was asked on many occasions, he/she could encode the stimuli and use a know rule.

Thus, the key to this distinction is whether the question has been asked repeatedly or can be expected (see Zentall, 2006). In the animal research cited (Babb \& Crystal, 2006; Clayton \& Dickinson, 1999a, 1999b), subjects are trained to answer an expected question. Thus, in the case of the scrub jays, the acquired rule might have been "if the memory (trace) of having cached worms is strong, look where you cached the worms, but if the memory of having cached worms is weak, look where you cached the peanuts."

Recently, Clayton and her colleagues (Clayton, Bussey, \& Dickinson, 2003; Clayton, Yu, \& Dickinson, 2003) have suggested two additional behavioral criteria that define episodic memory. In addition to the content of episodic memory (the what, where, and when), they proposed that episodic memory also has structure and flexibility. The term structure suggests that the three components of episodic memory - what, where, and when - form an integrated unit. That does seem reasonable for an episodic memory; however, how one would assess such a unit is not clear. The second criterion, flexibility, suggests that if one is given new information about the status of an outcome, one should be able to modify one's behavior accordingly (see Babb \& Crystal, 2006). Although flexibility can be considered an important cognitive ability, it is not clear how it is either a necessary or a sufficient condition for episodic memory.

What distinguished the present experiment (see also Zentall et al., 2001) from the others was the use of an unexpected question. On occasional probe trials, after choosing a line orientation after seeing the yellow or blue sample, the pigeon was asked, unexpectedly, if it could remember which side key it had just pecked. This is analogous to asking a person, "What did you have for breakfast this morning?" It is important that the question be unexpected not because expected questions cannot be answered using episodic memory, but because if the question is expected, one cannot exclude the use of semantic or rule-based memory to answer the question.

One further point needs to be made. One typically associates episodic memory with memory for an event that has taken place some time before. The question about breakfast this morning would usually be asked minutes or even hours after the event, and some episodes can be retrieved years after the event. But it is not clear in what sense this is a necessity. Imagine that I am about to leave the house and I notice that I do not have my glasses. In my mind's eye, I can retrace my steps and recall that just before I got ready to leave, I had answered the phone in the kitchen. Thus, I figuratively travel back in time, and I return to the kitchen to look for my glasses. Thus, to 
qualify as episodic, it should not be necessary to be able to retrieve distant memories. Our earlier research (Zentall et al., 2001) involved what was nominally a 0 -sec delay between the pecking and the not-pecking behavior that was retrieved. In the present experiment, almost $2 \mathrm{sec}$ elapsed, on average, between the to-be-retrieved response and the unexpected request to retrieve it, lending credibility to the hypothesis that memory was involved.

Although the results of the present experiment and those of Zentall et al. (2001) may not qualify as a demonstration of episodic memory in nonhuman animals as it occurs in humans, as we refine the conditions under which we can demonstrate declarative memory that likely cannot be accounted for using semantic or rule-based processes, we can approach the kind of evidence that will allow us to conclude that the ability to recover memories episodically does not inevitably distinguish humans from other animals.

\section{AUTHOR NOTE}

This research was supported by Grant MH 63726 from the National Institute of Mental Health. Correspondence concerning this article should be sent to T. R. Zentall, Department of Psychology, University of Kentucky, Lexington, KY 40506-0044 (e-mail: zentall@uky.edu).

\section{REFERENCES}

BABb, S. J., \& CRYstal, J. D. (2006). Discrimination of what, when, and where is not based on time of day. Learning \& Behavior, 34, 124-130.

Blough, D. S. (1959). Delayed matching in the pigeon. Journal of the Experimental Analysis of Behavior, 2, 151-160.

Clayton, N. S., Bussey, T. J., \& Dickinson, A. (2003). Can animals recall the past and plan for the future? Nature Reviews Neuroscience, 4, 685-691.

Clayton, N. S., \& Dickinson, A. (1999a). Memory for the content of caches by scrub jays (Aphelocoma coerulescens). Journal of Experimental Psychology: Animal Behavior Processes, 25, 82-91.

Clayton, N. S., \& Dickinson, A. (1999b). Scrub jays (Aphelocoma coerulescens) remember the relative time of caching as well as the location and content of their caches. Journal of Comparative Psychology, 113, 403-416.

Clayton, N. S., Yu, K. S., \& Dickinson, A. (2003). Interacting cache memories: Evidence for flexible memory use by Western scrub-jays (Aphelocoma californica). Journal of Experimental Psychology: Animal Behavior Processes, 29, 14-22.

Tulving, E. (1972). Episodic and semantic memory. In E. Tulving \& W. Donaldson (Eds.), Organization of memory (pp. 382-403). New York: Academic Press.

Tulving, E. (1985). How many memory systems are there? American Psychologist, 40, 385-398.

Urcuioli, P. J., \& DeMarse, T. (1994). On the relationship between differential outcomes and differential sample responding in matchingto-sample. Journal of Experimental Psychology: Animal Behavior Processes, 20, 249-263.

URCUIOLI, P. J., \& HoniG, W. K. (1980). Control of choice in conditional discriminations by sample-specific behaviors. Journal of Experimental Psychology: Animal Behavior Processes, 6, 251-277.

Zentall, T. R. (2006). Mental time travel in animals: A challenging question. Behavioural Processes, 72, 173-183.

Zentall, T. R., Clement, T. S., Bhatt, R. S., \& Allen, J. (2001). Episodic-like memory in pigeons. Psychonomic Bulletin \& Review, 8, 685-690

Zentall, T. R., \& Hogan, D. E. (1974). Abstract concept learning in the pigeon. Journal of Experimental Psychology, 102, 393-398.

(Manuscript received February 23, 2007; revision accepted for publication June 5,2007 .) 